\title{
Study on Kinetics of Protein Forward Extraction from Quinoa by AOT/ Isooctane Reverse Micelles System
}

\author{
Xiaoyong $\mathrm{Wu}^{1}$, Yanxia Sun ${ }^{1}$, Yujie Shi ${ }^{1,2}$, Yuan Tang ${ }^{1,2}$, Jijun Tian ${ }^{1,2}$ and Gang Zhao ${ }^{2, *}$ \\ ${ }^{1}$ College of Pharmacy and Biological Engineering, Chengdu University, Chengdu, 610106, China \\ ${ }^{2}$ Key Laboratory of Coarse Cereal Processing Ministry of Agriculture, Chengdu, 610106, China \\ ${ }^{*}$ Corresponding author
}

\begin{abstract}
In this paper, the kinetic process of extracting total protein from quinoa by AOT/ isooctane reverse micelles was systematically studied. The effects of extraction time, extraction temperature, particle size and oscillation rate on quinoa protein forward extraction rate were investigated respectively. Results showed that extraction of quinoa protein by AOT/ isooctane reverse micelles system was mainly controlled by internal diffusion, that is, the diffusion of protein from the interior of the particle to the surface was the control step of the reverse micelle extraction process. The extraction process can be explained by shrinking core model. The macroscopic dynamic equation of the protein forward extraction by the reverse micelles system was established and verified by extraction experiments at different temperatures. The experimental results indicated that the extraction process belonged to first order reaction and the apparent activation energy was $14.65 \mathrm{~kJ} / \mathrm{mol}$. The kinetic equation model could better describe the reverse micelle extraction process of quinoa protein and could provide important theoretical reference for the comprehensive development and utilization of quinoa protein in the future.
\end{abstract}

Keywords—quinoa; forward extraction; reverse micelle; kinetics

\section{INTRODUCTION}

Reverse micelle are agglomerates in which a surfactant is dissolved in a non-polar solvent[1,2]. In a reverse micelle solution, the hydrophobic groups of the surfactants come into contact with the solvent, and the hydrophilic groups face inward to form a polar core[3-4]. The core can solubilize water to form a "water pool", and biologically active substances such as proteins can be dissolved in the "pool" and be extracted. At present, researchers have focused on the extraction of biochemical substances from reverse micelle and have achieved remarkable results[5]. The extraction rate of biochemical substances has been significantly improved by selecting different reversed micelle systems or using auxiliary ultrasonic technology and supercritical technology in the extraction process[6]. It is generally believed that the process of mass transfer of solid protein dissolved in reversed micellar solution is the direct action of reversed micelle and proteins at the interface between the two phases[7], completing the mass transfer process, accompanied by the solubilization of water and ions. More studies had shown that alkaline extraction of protein in tomato seeds indicates that the diffusion process of the protein inside the solid was the decisive step for extraction[89]. However, this extraction system was quite different from the reversed micelle extraction system[10]. Therefore, the kinetics of reversed micelle extraction of solid proteins needs further study.

In recent years, more and more studies have shown that quinoa has been introduced all over the world and has become a research hot spot in crop and food fields[11]. Quinoa contains a lot of high quality protein easily absorbed by human body, and its protein content is much higher than that of conventional crops such as rice, barley and corn[12]. The protein of quinoa is mainly composed of albumin and globulin, and the content of gliadin and glutenin is low. Among them, quinoa albumin has the function of reducing weight and blood lipids[13]. However, few reports have been reported on the forward extraction kinetics of quinoa protein extracted from the solid liquid phase with the reverse micelle system[14-15].

In this paper, the factors and dynamic characteristics of the protein forward extraction were analyzed and investigated with high protein quinoa as raw materials. The kinetic equation was set up to provide theoretical support for the application of AOT/ isooctane reverse micelle extraction in the development of the quinoa protein resources.

\section{EXPERIMENTAL}

\section{A. Reagents and Materials}

Mature white quinoa seeds were supplied by Qinghai Golden Millet agricultural science and Technology Development Co., Ltd. The seeds were cleaned and preserved at low temperature until use. AOT (bis (2-ethylhexyl) sulfosuccinate sodium salt), isooctane, $\mathrm{KCl}, \mathrm{KH}_{2} \mathrm{PO}_{4}, \mathrm{Na}_{2} \mathrm{HPO}_{4}$ were all supplied by Merck (Darmstadt, Germany). Water was double distilled. Automatic Karl Fischer moisture analyzer, water bath constant temperature oscillator, automatic Kjeldahl apparatus, centrifuge, etc were used in the protein extraction experiment.

\section{B. Analysis of Main Ingredients of Quinoa Powder}

Protein content was determined by Kjeldahl apparatus[16]. Water content was determined by Karl Fischer moisture analyzer[17].

\section{Preparation of AOT/ Isooctane Reverse Micelle Solution}

A certain amount of AOT was placed into a conical flask and different volume of isooctane was added. AOT was completely dissolved by magnetic stirring until the solution was transparent to form different concentrations of AOT. $0.1 \mathrm{~mol} / \mathrm{L}$ $\mathrm{KCl}$ phosphate buffer was added into the solution, and the solution was fully oscillated at room temperature and centrifuged at $5000 \mathrm{r} / \mathrm{min}$ for $10 \mathrm{~min}$ or stand for $12 \mathrm{~h}$ to obtain 
the AOT/ isooctane reverse micelle solution. The water content of the reverse micelle solution was measured by Karl Fischer moisture analyzer.

\section{Protein Forward Extraction Experiment}

$20 \mathrm{~mL}$ reverse micelle solution was placed in a $100 \mathrm{~mL}$ conical bottle and added the quinoa powder into the bottle. Extraction was operated at a certain time in a water bath oscillator at a certain speed, then centrifuged at 5000r/min for 10 minutes. The system was divided into two layers. The protein concentration in the supernatant was measured by Kjeldahl method.The protein extraction rate was calculated as follows: $R_{e}={ }^{C_{r}} / C_{t} \times 100 \%$.

In the formula, $R_{e}$ was the protein forward extraction rate; $C_{r}$ was the protein concentration in reverse micelle solution $(\mathrm{mg} / \mathrm{ml}) ; C_{t}$ was the total protein concentration in quinoa (mg/ml).

\section{E. Selection of Kinetic Model of Reverse Micelle Extraction}

The process of extracting quiona protein by reversed micelle may include four steps[18]: 1) The reverse micelle diffuse from the main body of the organic phase to the solid-liquid interface; 2) The proteins diffuse from the solid interior to the solid-liquid interface; 3) The proteins in the solid-liquid interface and the reverse gum The beam collides to form a reversed micelle containing the protein; 4) The reversed micelle containing the protein diffuses from the two-phase interface into the organic phase. The extraction process may include 4 kinds of resistance control: external diffusion resistance control, interfacial reaction resistance control, internal material diffusion resistance control and mixed resistance control.

For the liquid-solid non-catalytic reaction model, the most widely used was the shrinking core model[19]. In deriving the reaction rate equation, the model needs to do three assumptions: 1 ) the reaction occurs on the apparent interface of the unreacted core; 2 ) the reaction process is a quasi steady state process; 3 ) the temperature in the solid is homogeneous and the reaction is a pseudo first-order irreversible reaction[20-21].

With reference to this model, the liquid-solid extraction mathematical model of quinoa protein extraction by AOT/ isooctane can be expressed as[22]:

$$
t=\frac{1}{k_{1}} x+\frac{1}{k_{2}}\left[1-(1-x)^{1 / 3}\right]+\frac{1}{k_{3}}\left[1+2(1-x)-3(1-x)^{2 / 3}\right]
$$

In the equation: $t$ is the extraction time; $x$ is the protein front extraction rate; and $k_{1}, k_{2}, k_{3}$ are the constant of the property of the material system.

If the extraction process was controlled by external diffusion, the equation (1) can be simplified to

$$
t=x / k_{1}
$$

If the extraction process was controlled by interface, the equation (1) can be simplified to

$$
t=\frac{1}{k_{2}}\left[1-(1-x)^{1 / 3}\right]
$$

If the extraction process was controlled by internal diffusion, the equation (1) can be simplified to

$$
t=\frac{1}{k_{3}}\left[1+2(1-x)-3(1-x)^{2 / 3}\right]
$$

When reaction was controlled by both interface chemical reaction and internal diffusion, named mixed control , the equation (1) was simplified to:

$$
t=\frac{1}{k_{2}}\left[1-(1-x)^{1 / 3}\right]+\frac{1}{k_{3}}\left[1+2(1-x)-3(1-x)^{2 / 3}\right]
$$

\section{RESULTS AND DisCUSSIONS}

\section{A. Analysis of Main Ingredients of Quinoa Powder}

The moisture content of the quinoa powder was $6.88 \%$ and the protein content was $21.6 \%$.

\section{B. Effect of Extraction Time}

Figure 1 showed that increasing the extraction time can promote the protein to enter the reverse micelle solution. Between 20-60 min, the forward extraction rate of protein increased with the extraction time, and the extraction rate of protein increased slowly after $60 \mathrm{~min}$, indicating that the process of forward extraction was close to balance at $60 \mathrm{~min}$.

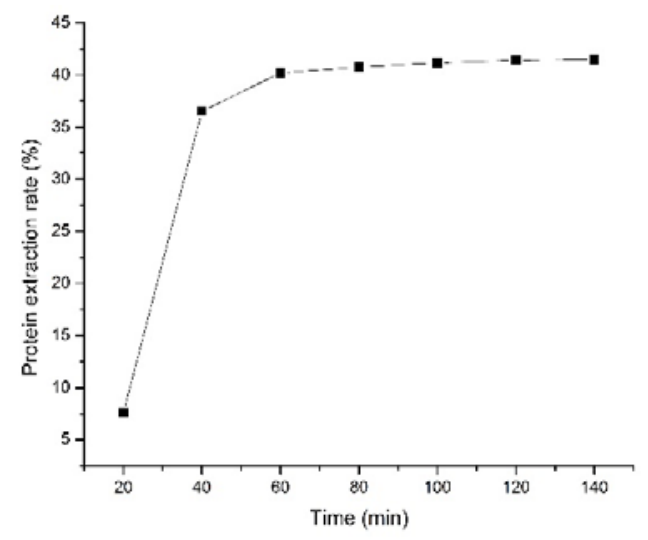

FIGURE I. EFFECT OF EXTRACTION TIME ON PROTEIN EXTRACTION RATE

\section{Effect of Temperature on Protein Extraction Rate}

At the same extraction time, the higher the temperature, the higher the extraction rate; at the same temperature, with the increasing of extraction time, the extraction rate of protein also gradually increased (Table 1). However, during the actual experiments, we found that the forward extraction rate of protein at $50^{\circ} \mathrm{C}$ was lower than that of $40^{\circ} \mathrm{C}$. Temperature had two main reasons on protein extraction[23]. First, when the temperature increased, the viscosity of the system decreased, which reduces the diffusion resistance of the molecules and increased the mass transfer rate, whereas the extraction rate 
decreases. Second, when the temperature was too high, some protein denature leads to the decrease of solubility in water. At the same time, the increase of temperature might also affect the stability of the reverse micelle system, which leads to the decrease of protein extraction rate. So, the extraction temperature range was selected $25-40{ }^{\circ} \mathrm{C}$ to achieve good extraction results.

TABLE I. THE FORWARD EXTRACTION RATE OF PROTEIN AT DIFFERENT TEMPERATURES AND TIMES

\begin{tabular}{ccccc}
\hline \multirow{2}{*}{ Time (min) } & \multicolumn{4}{c}{ Protein extraction rate(\%) } \\
\cline { 2 - 5 } & $25^{\circ} \mathrm{C}$ & $30^{\circ} \mathrm{C}$ & $35^{\circ} \mathrm{C}$ & $40^{\circ} \mathrm{C}$ \\
\hline 10 & 21.49 & 24.93 & 28.33 & 29.73 \\
20 & 30.45 & 33.19 & 35.24 & 38.79 \\
30 & 38.57 & 41.95 & 42.91 & 46.61 \\
40 & 44.14 & 49.17 & 48.79 & 51.72 \\
50 & 51.34 & 53.48 & 54.98 & 57.33 \\
60 & 55.01 & 57.09 & 58.42 & 61.51 \\
70 & 57.67 & 60.02 & 62.53 & 64.42 \\
80 & 59.91 & 62.65 & 65.79 & 68.26 \\
\hline
\end{tabular}

The above data were substituted into the equation (3) and (4) to examine whether the extraction was controlled by internal diffusion or interface reaction. The results were shown in Figure 2 and 3. Results showed that in the extraction process, the reaction time was linearly related to $1+2(1-x)-3(1-x)^{2 / 3}$ and passed through the origin; Figure 3 showed that the extraction time did not have a linear relationship with $1-(1-x)^{1 / 3}$ and there was no regularity, so it was not possible to have a linear relationship with $\frac{1}{k_{2}}\left[1-(1-x)^{1 / 3}\right]+\frac{1}{k_{3}}\left[1+2(1-x)-3(1-x)^{2 / 3}\right]$. This indicated that the extraction process did not belong to the mixed control process[24]. So it can be proved from Figure. 2 and 3 that the process which quinoa protein extracted by reverse micelle was controlled by internal diffusion.

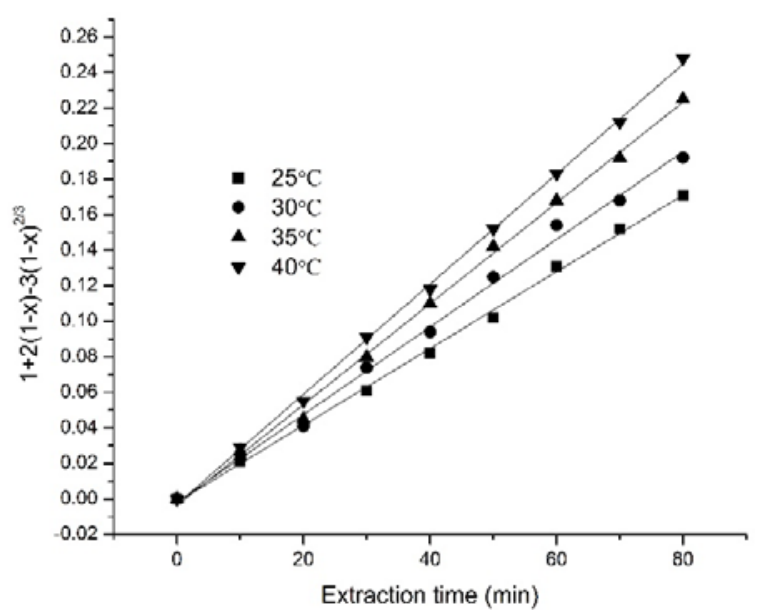

FIGURE II. THE RELATIONSHIP BETWEEN EXTRACTION TIME AND $1+2(1-x)-3(1-x)^{2 / 3}$ AT DIFFERENT TEMPERATURES

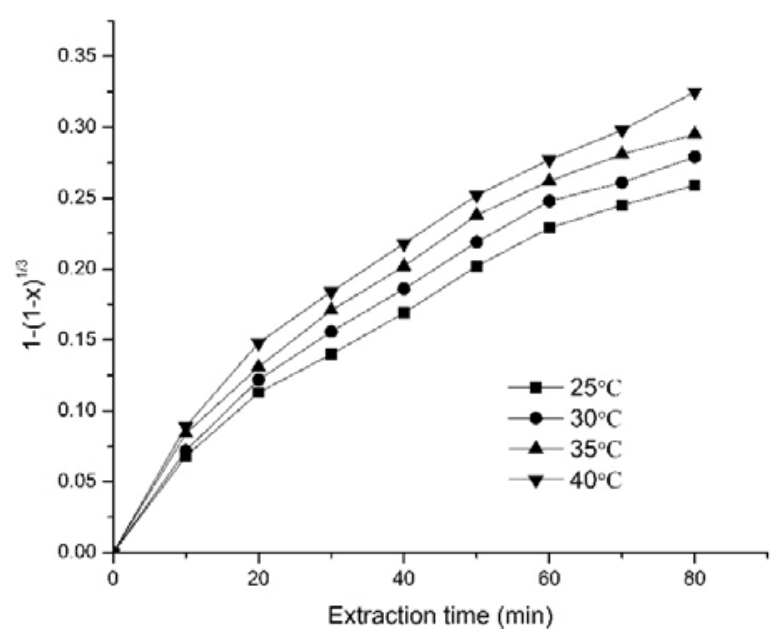

FIGURE III. THE RELATIONSHIP BETWEEN EXTRACTION TIME

$$
\text { AND } 1-(1-x)^{1 / 3} \text { AT DIFFERENT TEMPERATURES }
$$

\section{The Effect of Particle Size on Protein Extraction}

As shown in Figure 4, the Figures were all straight and all pass through the origin. It indicated that at the same extraction time, the smaller the particle size, the higher the protein extraction rate. This result could be explained in two aspects[25]. Firstly, the reduction in particle size increases the specific surface area of the particles which results in a larger mass transfer surface area of the quinoa powder. Secondly, under the premise of a certain total quality, the smaller the particle size, the more the number of particles, so that the larger the reaction area, the faster the leaching rate. The reduction of the particle diameter shortened the diffusion distance of the protein inside the particle and reduced the internal diffusion resistance of the protein, thereby increasing the mass transfer rate[26].The results showed that in the forward extraction, the diffusion of proteins inside the particles had a significant effect on the extraction effect.

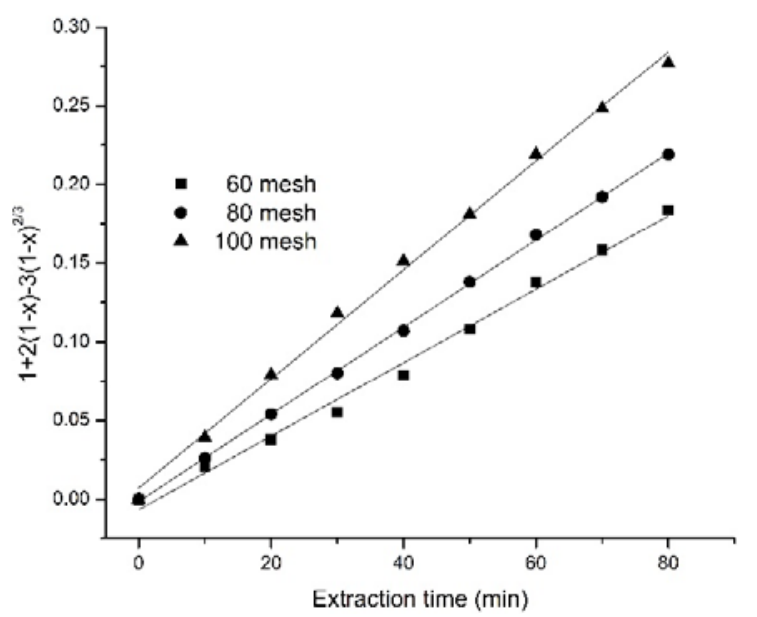

FIGURE IV. EXTRACTION KINETICS CURVES AT DIFFERENT PARTICLE SIZES 


\section{E. Effect of Oscillation Rotation Speed on Protein Extraction Rate}

Figure 5 showed that when the oscillating speed was less than $160 \mathrm{r} / \mathrm{min}$, the extraction rate of protein increased with the increase of the oscillating speed; when the oscillating speed was greater than $160 \mathrm{r} / \mathrm{min}$, the extraction rate of protein was no longer changed. Oscillation could enhance diffusion, promoted well dispersion of reversed micelle, and promoted efficient diffusion of liquid reactants through the solid product layer[27], thereby increasing the area of the phase boundary and the diffusion coefficient. As the oscillation rate increased, the reverse micelle were fully contacted with quinoa powder, and the effective mass transfer area increased, leading to a significant increase in the extraction rate. When the oscillation rate was greater than $160 \mathrm{r} / \mathrm{min}$, the extraction rate had no obvious change, which indicated that the external diffusion had little effect on protein extraction.

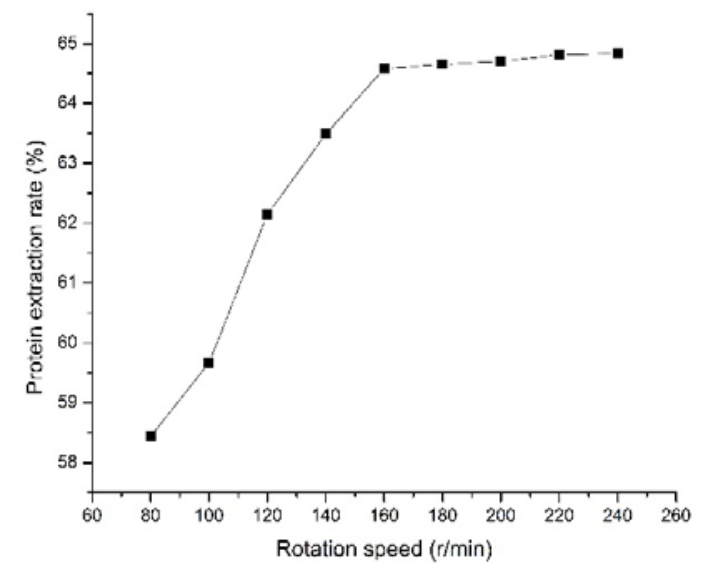

FIGURE V. EFFECT OF EXTRACTION TIME ON PROTEIN EXTRACTION RATE

\section{F. Effect of AOT Concentration on Protein Extraction Rate}

Substituted the results of protein extraction at different AOT concentrations into the equation (4) and made the dynamic curve, the results were shown in Figure 6. It showed that extraction time and $1+2(1-x)-3(1-x)^{2 / 3}$ had a linear relationship and passed through the origin, indicating that the extraction of quinoa protein from reverse micelle was controlled by internal diffusion[28]. At the same time, with the increase of AOT mass concentration, the protein extraction rate gradually increased. The low surfactant concentration was detrimental to the formation of reversed micelle, resulting in a low protein extraction rate. Increasing the mass concentration of AOT will increased the concentration of reversed micelle in the solution and the amount of solubilized water in the "pool" will increased accordingly. If the AOT concentration was too high, the viscosity of the system will increased, resulting the decrease of the intermolecular collision between micelle and the extraction rate of protein. It also leads to the complexity of the back extraction process in the future[29]. Through repeated experiments, the maximum concentration of AOT chosen was $0.1 \mathrm{mg} / \mathrm{ml}$ to obtain better protein extraction rate.

The apparent rate constant $k_{3}$ were obtained according to the extraction kinetic curve at different AOT concentrations. Take $\log \mathrm{C}$ as the abscissa and $\log k_{3}$ as the ordinate, as shown in Figure 7. The regression equation was linear $(y=0.9409 x-1.859)$ and the $\mathrm{R}^{2}=0.999$. It indicated that the reaction order in the quinoa protein extraction process can be regarded as a firstorder reaction[30].

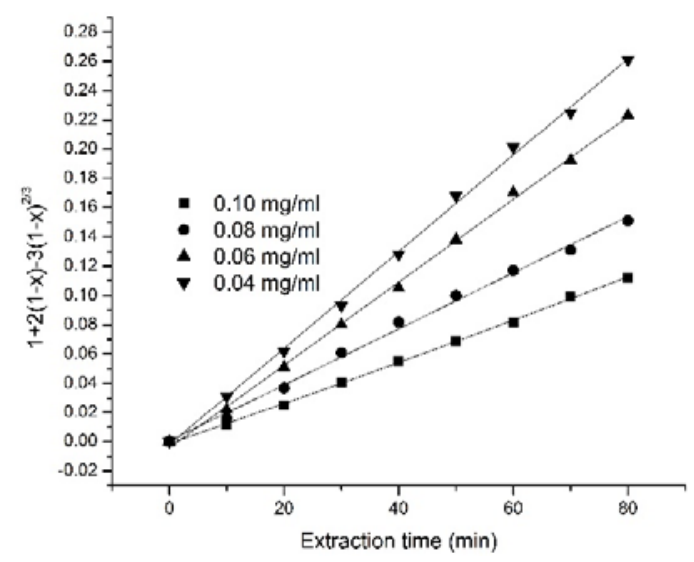

FIGURE VI. EXTRACTION KINETIC CURVES AT DIFFERENT AOT CONCENTRATIONS

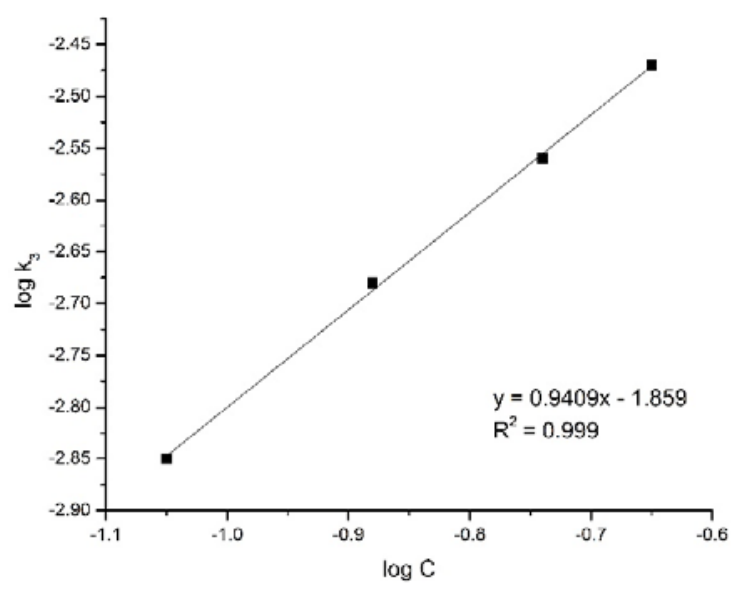

FIGURE VII. THE PLOT OF LOG K3 VERSUS LOG C

\section{G. Establishment of Reverse Micelle Extraction Kinetic Equation}

Results from Figure 4 and Figure 5 indicated that the effect of external diffusion on protein extraction was very small in the process of quinoa protein extraction. The diffusion of proteins inside the particles had a significant effect on the extraction of proteins. Therefore, it inferred that during protein forward extraction, the diffusion of protein from the inside of the particle to the surface of the particle was a control step of the protein extraction, so the extraction process belong to internal diffusion control. Therefore, the kinetics of extraction process can be explained by the shrinking core model.

According to Arrhenius formula $k_{3}=k_{0} e^{-E a / R T}$, plotted $\ln k_{3}$ versus $1 / \mathrm{T}$. The following data obtained from Figure 8 that $E a / R=1761.8$, apparent activation energy $E a=14.65 \mathrm{~kJ} \cdot \mathrm{mol}^{-1}$, it indicated that the process of extracting quinoa protein from reverse micelle belong to internal diffusion control. The kinetic equation of extracting quinoa protein from reverse micelle can 
be further expressed as: $k_{0} e^{-1761.8 / T} \times t=1+2(1-x)-3(1-x)^{2 / 3}$ (named equation 6), where $k_{0}$ was a temperature-independent constant. $k_{0}=1.22$ was obtained by calculation by substituting the protein extraction rate at different temperature into the equation (6).

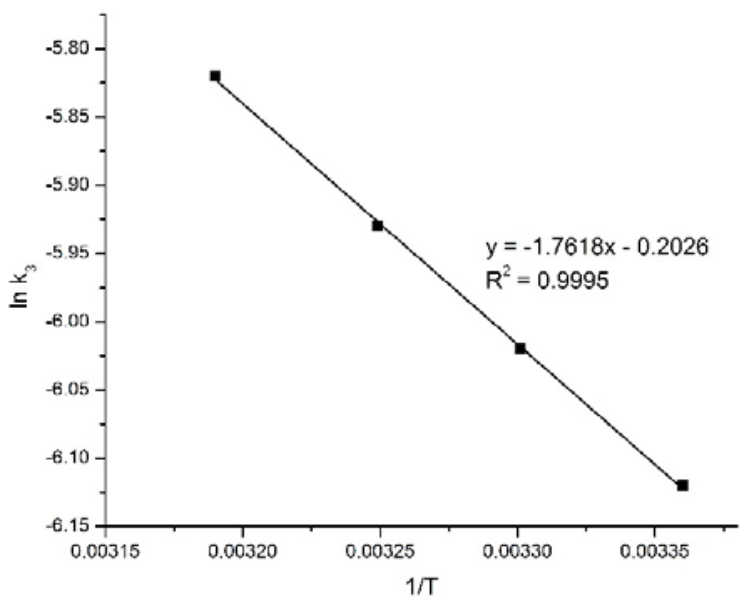

FIGURE VIII. THE PLOT OF LN K3 VERSUS 1/T

In summary, the kinetic equation for reversed micelle extraction of quinoa protein can be expressed as:

$$
1.22 e^{-1761.8 / T} \times t=1+2(1-x)-3(1-x)^{2 / 3} .
$$

\section{H. Verification of Kinetic Equation}

The kinetic equation was verified by the quinoa protein extraction data by reverse micelle at 20, 26, 32 and $45{ }^{\circ} \mathrm{C}$ respectively. Results were shown in Figure 9. The test results were in good agreement with the experimental values. Therefore, it showed that the shrinking core model can be better applied to the kinetics of quinoa protein extracted by AOT/ isooctane reverse micelle system.

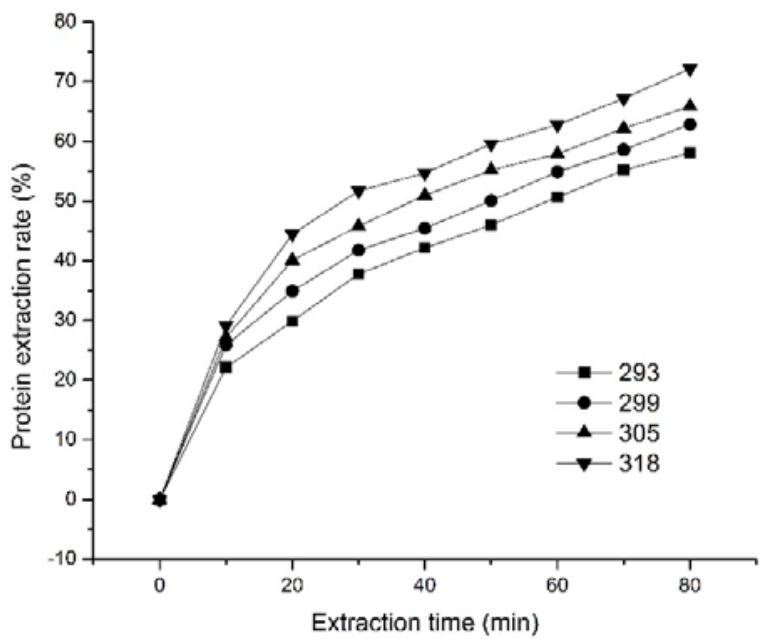

FIGURE IX. RELATIONSHIP BETWEEN THE EXTRACTION RATE AND THE EXTRACTION TIME

\section{CONCLUSION}

The process of extraction of quinoa protein by reverse micelle was studied and the factors affecting the extraction of quinoa protein were investigated. The results showed that higher temperature, smaller particle size and higher reverse micelle concentration were beneficial to the internal diffusion reaction. The experimental results showed that the solid-liquid reaction was a first-order reaction with respect to the concentration of reversed micelle, and the apparent activation energy under the experimental conditions was $14.65 \mathrm{~kJ} \cdot \mathrm{mol}^{-1}$. During the extraction process of AOT/ isooctane system, the diffusion of protein from the interior of the particle to the particle surface was the control step of mass transfer. The mass transfer resistance of the protein into the polar core of the reverse micelle and the external diffusion can be ignored. The process can be simulated by the shrinking core model. The macroscopic kinetic equation of the quinoa protein extracted by AOT/ isooctane reverse micellesystem was: $1.22 e^{-1761.8 / T} \times t=1+2(1-x)-3(1-x)^{2 / 3}$.

\section{ACKNOWLEDGMENTS}

This work was supported by the Key Laboratory of Coarse Cereals Processing, Ministry of Agriculture, Chengdu University (No. 2017Y0012). Xiaoyong Wu and Yanxia Sun authors contributed equally to this work.

\section{REFERENCES}

[1] M.M.Cardoso, R.M.C. Viegas, J.P.S.G. Crespo, Chemical Engineering Science, 5,2835-2847(2000).

[2] Eskici, Gözde1;Axelsen, Paul H, J Phys Chem B,44, 11337-11347(2000).

[3] Puspal Mukherjee, Shradhey Gupta,Shahnawaz Rafiq, Langmuir, 7, 1693-1699(2016).

[4] Luna,M. Alejandra1;Correa,N. Mariano1;Silber, Juana J, Journal of Physical Organic Chemistry, 11, 580-585(2015).

[5] Gaikaiwari Raghavendra P, Wagh Shipa A, Kulkarni Bhaskar D,Bioresource Technology, 108, 224-230.

[6] Hemavathi A B, HebbarH Umesh, Raghavarao K S M S, Separation \& Purification Technolog, 2, 263-268(2010).

[7] Liu Jun -Guo, Xing Jian -Min, Rui Shena, Biochemical Engineering Journal, 3, 273-278(2012).

[8] M Mechmeche, F Kachouri, M Chouabi, Food Analytical Methods,111(2016).

[9] X Shen, Food Industry, 7,107-109(2005).

[10] K He, Z Zou, Y Hu, Journal of Separation Science, 4, 703(2016).

[11] SE Jacobsen, Food Reviews International, 1, 167-177(2003).

[12] SE Jacobsen, Food Reviews nternational,19:176(2003).

[13] C Vilche, M Gely, E Santalla,Biosystems Engineering, 1, 59-65(2003).

[14] M Föste, D Elgeti, AK Brunner, Food \& Bioproducts Processing, 96, 20-26(2015).

[15] MR Guerreo-Ochoa, R Pedreschi, R Chirinos, International Journal of Food Science \& Technology, 8, 1815-1822(2015).

[16] H Wang, N Pampati, WM Mccormick, Journal of Pharmaceutical Sciences, 6,1851-1857(2016)

[17] EC Milberger, K Uhrig, HC Becker, Analytical Chemistry,10, 11921194(2002).

[18] MJ Pires, MR Aires-Barros, JMS Cabral, Biotechnology Progress, 3, 290 301(2010).

[19] J Blamey, M Zhao, V Manovic, Chemical Engineering Journal, 291, 298305(2016).

[20] AK Sadhukhan, P Gupta, RK Saha, Fuel, 1, 162-169(2010).

[21] R López-Fonseca, JR González-Velasco, JI Gutiérrez-Ortiz, Chemical 
Engineering Journal, 2, 287-294(2009).

[22] AK Sadhukhan, P Gupta, RK Saha, Fuel, 1, 162-169(2010).

[23] M Hentsch, P Menoud, E Flaschel, Biotechnology Techniques, 5, 423428(1992).

[24] HS Joo, CG Kumar, GC Park, Process Biochemistry, 11, 14411447(2004).

[25] Kumoro, Retnowati, DS Budiyati, Reaktor, 2, 124-130(2015).

[26] TA Russin, Y Arcand, JI Boye, Journal of Food Processing \& Preservation, 3, 308-319(2010).

[27] A Neufeld-Cohen, MS Robles, R Aviram, Proceedings of the National Academy of Science, 12, E1673(2016).

[28] N Banno, Y Miyamoto, K Tachikawa, IEEE Transactions on Applied Superconductivity, 3, 1-4(2016).

[29] BC Ayala, AJ Gallardo, AM Olivares, Journal of Information Systems and Technologies, 24,1-12(2017).

[30] H Suk, Advances in Water Resources, 94, 412-423(2016). 